\title{
Control Experimentation for Undergraduate Students
}

\author{
Chetan Chandrasekara, Asad Davari, Senior Member, IEEE \\ West Virginia University Institute of Technology, Montgomery West Virginia 25136
}

Keywords: Control Laboratory, Education, Linear System

\begin{abstract}
This paper describes Automatic Control Laboratory experiments developed at the Department of Electrical and Computer Engineering at the West Virginia University Institute of Technology. Significance is given to unstructured based instruction. The Laboratory course is offered concurrently with an introductory lecture course in Automatic Control Theory. Experiments are designed to give students thorough understanding of basic concepts and phenomenon of control theory.
\end{abstract}

\section{INTRODUCTION}

Control system engineering is highly multidisciplinary branch of engineering. In fact, control system engineering is a part of many branches of engineering. Teaching control system can be both conceptual and experimental. Control system experimentation, however, is not an established discipline, but there are many fundamental issues that are worthy of deep and careful consideration. This paper is an integration of many recommendations on enhancing the undergraduate education. In general educational laboratory exercises can be in general from programming highly structured tasks to unstructured tasks [3]. This paper draws attention to unstructured experimentation. In this approach, the students are given a statement of objectives with an outline of steps. There are many appropriate procedures that students can follow.

Control system education can be enhanced by employing some of the ideas cited by [10] such as use of different analogies, dimensions and units, emphasis on system identification, unexpected system changes, emphasis on sensors and actuators, emphasis on control objectives, physics behind the control law, build a simple control system and importance of feedback in a control system.

Technology is a critical tool to support, enhance teaching and research. Here at the Department of Electrical \& Computer Engineering (ECE Dept), West Virginia University Institute of Technology (WVU TECH), emphasis is given for technology as a tool for education as well as research [1].

The automatic control laboratory course at ECE Dept, WVU TECH, is designed to supplement the study of Automatic Control Theory as presented in the undergraduate course. The laboratory experiments have also addressed many issues such as controller physics, controller architecture, controller hardware and controller tuning. This paper also describes the utilization of the laboratory tools for different laboratory experiments for both the undergraduate and graduate students.

\section{AUTOMATIC CONTROL LABORATORY}

The topics introduced in the course ELCE 424 Automatic Control such as introduction to control systems, time domain analysis, control system characteristics and performance of feedback control, complement the laboratory course. The course, entitled "Automatic Control Laboratory," is organized around consideration of principles of control systems. There are many aspects of a Control System problem that can be investigated. For example, formulation of a mathematical model for the 'Plant,' or the system to be controlled, and identification of values of the parameters of this mathematical model is seldom given much attention. The emphasis in the laboratory exercises is on plant identification, open-loop and closed loop characteristics, effect of damping and design, and implementation of PID control schemes. There are numerous aspects of control system that present difficulties in the laboratory not apparent from classroom analysis. It is the intent of the laboratory to allow the students to investigate such problems and give them an opportunity to solve such practical problems of control system engineering. The laboratory experiments are designed to provide insight into the control system principles through theoretical as well as practical demonstration and experimentation. The laboratory experiments are designed in such a way that the theoretical principles can be demonstrated.

\section{STRUCTURE OF THE LABORATORY}

There are four vital Educational Control tools employed. They are ECP Model 205 (Torsional Control System), [6], ECP Model 210 (Rectilinear Control System), [5], ES151 (Educational Servo), [15] and SFT154 (Educational Servo Trainer), [14]. These systems are designed to provide insight to control system principles through hands-on demonstration and experimentation. The electromechanical 
plant has a full complement of control hardware and software. The user interface to the system is via a friendly, versatile, PC window environment which supports a broad range of controller specification, trajectory generation, data acquisition, and plotting features. The systems are designed to accompany introductory through advanced level controls courses and support either high level usage (i.e. direct controller specification and execution) or detailed userwritten algorithms [7].

The electromechanical apparatus may be transformed into a variety of dynamic configurations which represent important classes of "real life" systems. The ECP Model 205 (Torsional Control System) and ECP Model 210 (Rectilinear Control System) apparatus represents many such physical plants including rigid bodies; flexibility in linear drives, gearing and belts; and coupled discrete vibration with actuator at the drive input and sensor collocated or at flexibly coupled output (noncollocated). Thus the plant models may range from a simple double integrator to a fourth order case with two lightly damped poles with either two or no zeros.

The experimental control system is comprised of the three subsystems. The first of these is the electromechanical plant which consists of the spring/mass mechanism, its actuator and sensors. The design features a brushless DC servo motor, high resolution encoders, adjustable masses, and reconfigurable plant type.

The second subsystem is the real-time controller unit which contains the digital signal processor (DSP) based real-time controller, servo/actuator interfaces, servo amplifier, and auxiliary power supplies. The DSP is capable of executing control laws at high sampling rates allowing the implementation to be modeled as being continuous or discrete in time. The controller also interprets trajectory commands and supports such functions as data acquisition, trajectory generation, and system health and safety checks. A logic gate array performs motor commutation and encoder pulse decoding. Two optional auxiliary digital-to-analog converters (DAC's) provide for real-time analog signal measurement. This controller is representative of modern industrial control implementation. The third subsystem is the executive program which runs on a PC under the DOS or Windows operating system. The ECP Executive program is the user's interface to the system. It is a menu driven / window environment that the user will find is intuitively familiar and quickly learned. This software runs on a PC and communicates with ECP's digital signal processor (DSP) based real-time controller. Its primary functions are supporting the downloading of various control algorithm parameters (gains), specifying command trajectories, selecting data to be acquired, and specifying how data should be plotted. In addition, various utility functions ranging from saving the current configuration of the Executive to specifying analog outputs on the optional auxiliary DAC's are included as menu items.

The User Units dialog box provides the user with various choices of angular or linear units. For ECP Model 205 the choices are counts, degrees and radians. There are 16000 counts, 360 degrees and $2 \pi$ radians per revolution of both the load and drive inertia disks. And for ECP Model 210 the choices are counts, centimeters and inches. There are 1604 counts, per centimeter travel of the mass carriages. By clicking on the desired radio button the units are changed automatically for trajectory inputs as well as the Background Screen displays, plotting and jogging activities.

Setup Control Algorithm allows the entry of various control structures and control parameter values to the realtime controller, Fig 1. The currently available feedback options are:

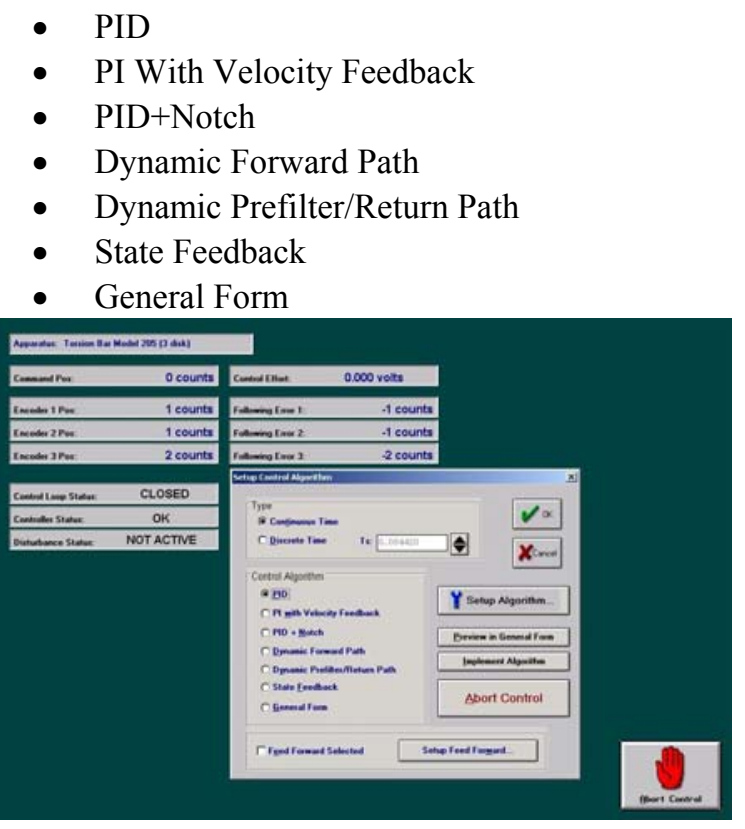

Fig 1 Set Up Control Algorithm Dialog Box

The Feedback ES151 Educational Servo System is a high quality electro-mechanical servomechanism. The system has two basic subsystems,

- The Actuator Unit, and

- The Educational Servo.

The Actuator Unit

The Actuator Unit has the following components

- A 24V de electric motor whose nominal full speed is $3000 \mathrm{r} / \mathrm{min}$.

- A tachogenerator is a device which generates a voltage related to speed.

- The gearbox is a worm and wheel is used to drive an output shaft, the speed of which is only $1 / 30$ th of that of the motor shaft and at right angles to it. Full output speed is about $100 \mathrm{r} / \mathrm{min}$. 
- The output disc and potentiometer is calibrated to indicate output shaft position (angular) and has stroboscope tracks that can be used to determine certain speeds. In front of the disc is a SENS0R or TRANSDUCER called a POTENTIOMETER which will give a voltage output that is related to the shaft position.

- A Loading Unit is used in conjunction with the large thin aluminum disc supplied in the accessory pack.

In use, the disc is mounted on the motor shaft and then runs between the magnets that can be positioned, using the lever, to cover a large or small area of the disc. This position is calibrated 0 to 10 on a scale at the side of the unit.

When the disc rotates between the magnets a torque is generated, due to what are called Eddy Currents, which tries to slow the motor. The braking effect depends on the motor speed and the amount of magnet overlap on the disc.

\section{The Educational Servo}

The single 'control box' actually contains several units, the details of which will be considered later. To emphasize the main function of this item it will be, in future, referred to in the text as the Control Unit. It contains all the necessary power supplies for the system to operate and must be coupled to an electrical power source of $100-240 \mathrm{~V}$ ac as required. The unit is fused and if necessary the fuse can be replaced by pressing and turning the fuse holder.

The SFT154 Servo Fundamentals Trainer is intended to provide students with a sound introduction to the principles of servomechanisms, and by extension to those of closed-loop systems more generally. It has facilities for examining a system based entirely on analogue signals and also a system which is almost entirely digital. The SFT154 Servo Fundamentals Trainer consists of 3 units:

- Analogue Unit AU 154A,

- Digital Unit DU1548, and

- Mechanical Unit MU 154C.

The Analogue Unit and the Digital Unit are separate, allowing a purely analogue system to be converted into a analogue and digital system.

\section{Analogue Unit}

The Analogue Unit connects to the Mechanical Unit through a 34-way ribbon cable which carries all power supplies and signals enabling the normal circuit interconnections to be made on the Analogue Unit using the $2 \mathrm{~mm}$ patching leads provided. The unit enables a basic system to be configured and contains facilities to introduce compensation to investigate improvement in overall system performance.

\section{Digital Unit}

The Digital Unit connects to the Mechanical Unit through a 34-way ribbon cable carrying all power supplies and signals enabling the normal circuit interconnections to be made on the unit using the $2 \mathrm{~mm}$ patching leads provided.

The unit contains an A/D converter to enable continuous input or error signals to be digitized for computer input. The computer input can also be obtained directly from the output transducer or bi-phase speed tracks. The computer output can be converted to a continuous motor drive signal through a D/A in the unit or to a Pulse Width Modulation (PWM) switching signals for motor drive.

\section{Mechanical Unit}

The Mechanical Unit contains a power amplifier to drive the motor from an analogue or switched input. The motor drives the output shaft through a 32:1 belt reduction. The motor shaft also carries a magnetic brake disc and an analogue speed transducer (tachogenerator). A two-phase pulse train for digital speed and direction sensing is also derived from tracks on the brake disc.

The output shaft carries analogue (potentiometer) and digital (64 location Gray code) angle transducers. The unit contains a simple signal generator to provide low frequency test signals, square and triangular waves, and requires an external power supply providing:

$+15 \mathrm{~V}, 0,-15 \mathrm{~V}$ at $1.5 \mathrm{~A},+5 \mathrm{~V}, 0$, at $0.5 \mathrm{~A}$, The Feedback PS 446 is suitable.

\section{CLASSIFICATION OF EXPERIMENTS}

This section describes the experiments that reinforce the principles of analyzing a simple control system. The experiments are as follows:

- Motor characteristics

- Open loop and closed loop analysis

- System identification

- Effect of damping

- Effect of Proportional, Integral and Derivative action on a given system.

- Project

The first two experiments are structured, in such a way that students are given step by step instructions. The objective of the first laboratory exercise is to learn the steady state characteristics of the system and observe the changes in the output of the system with changing load conditions. The second exercise demonstrates the significance of feedback in a control system. An Open loop system is a system in which the output is neither measured nor fed back for comparison with the input. Thus, each reference input corresponds to a fixed operating condition. As a result, the accuracy of the system depends on calibration. Closed loop system maintains a prescribed relationship between the output and the reference input by comparing the difference as a means of control. The aim of the experiment is to control the position of the output potentiometer. 
The next experiment outlines instructions for mathematical modeling of a simple second order system. After completing the exercises the students will:

- Be familiar operating the ECP system operation

- Be able to recognize the hardware/software settings

- Be able to calculate the hardware gains and develop a transfer function by measuring plant parameters i.e., spring and damping constants.

This exercise gives a procedure for identifying the plant parameters. The approach will be to use certain fundamental properties of lightly damped second order system to indirectly measure the inertia, spring and damping constants of the plant by making measurements of the plant while setup in a pair of classical spring-mass configuration.

The progressive decay of the response of the system with time in the amplitude of the free oscillations of the system may be termed as the damping of the system. The next exercise outlines instructions to investigate the damping in a second order system.

After completing the exercise the students will:

- Understand the differences between under damped, critically damped, and over damped systems.

- Investigate the effect of changing inertia, stiffness of the spring on the damping of a second order spring-mass-damper system.

- Understand various ways of applying as well as changing the damping of a given system.

In the next exercise the effect of Proportional, Integral and Derivatives actions on the system performance are investigated. These exercises should give the students a thorough understanding of the three terms of PID controller.

After completing the exercises the students will be able:

- To investigate the effects of Proportional, Integral and Derivative control action on the system performance

- To analyze the advantages and disadvantages of each of the control actions.

The last exercises are projects given to the students to demonstrate their ability to analyze a given system and design as well as implement a PID control scheme. Students will be able comment on the stability of the system (Open loop response) and which control scheme is best suitable for the given system.

\section{EXPERIMENTAL RESULTS}

This section illustrates some of the experimental results obtained by the students.

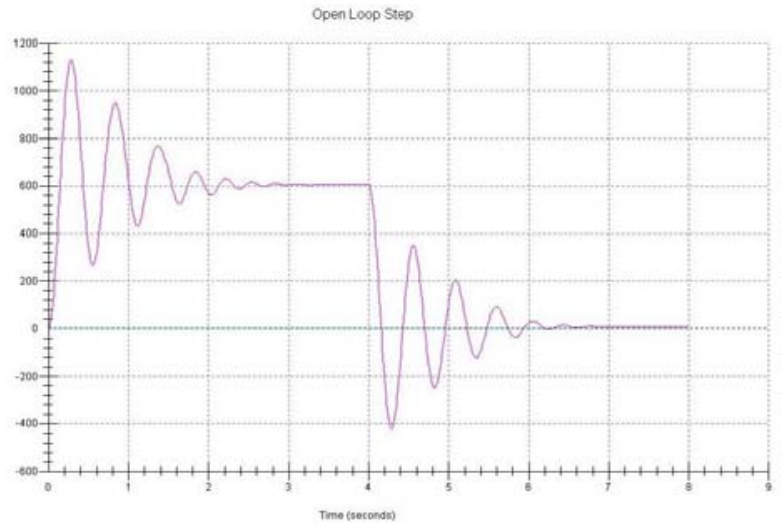

Fig 4 Open Loop Response on ECP Model 205 Torsion System

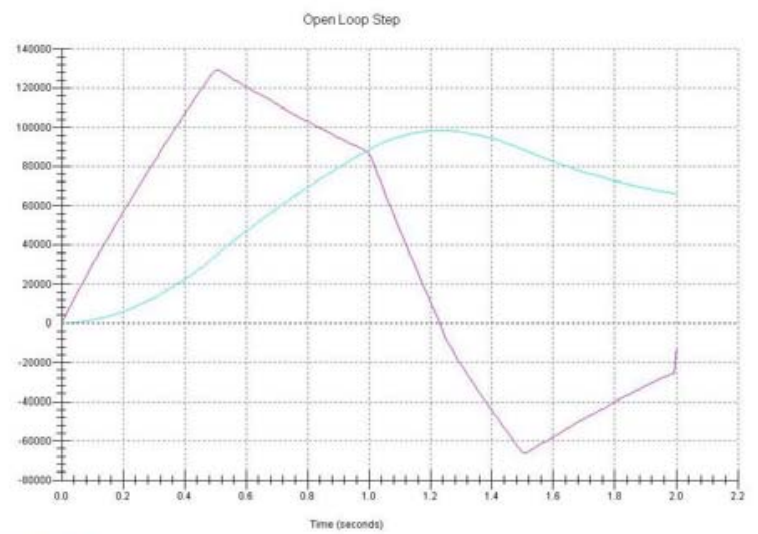

Fig 5 Open Loop Velocity profile of the base disk

The objective of this laboratory exercise is to identify the plant parameters. The approach will be to use certain fundamental properties of lightly damped second order system to indirectly measuring the inertia, spring and damping constants of the plant by making measurements of the plant while setup in a pair of classical spring-mass configuration. Fig 4 shows the oscillation of the system with the masses attached to the disk. The amplitude of oscillation helps in calculating the natural frequency and damped natural frequency of the system. The frequencies are estimated by logarithmic decrement of the oscillations. A number of trials can be performed in each of the cases to get consistent values. Fig 5 shows the velocity profile of the disk, which is useful in measuring the acceleration and deceleration of the system. These values are measured in turn to calculate the hardware gain of the system.

The objective of this exercise is to observe the effects of Proportional, Integral, Derivative actions individually as well as combination of two components on the system. A proportional controller $\left(\mathrm{K}_{\mathrm{p}}\right)$ will have the effect of reducing the rise time, but difficult to eliminate the steady-state error. An integral control $\left(\mathrm{K}_{\mathrm{i}}\right)$ will have the effect of eliminating the steady-state error, but it may make the transient response worse. A derivative control $\left(\mathrm{K}_{\mathrm{d}}\right)$ will 
have the effect of increasing the stability of the system, reducing the overshoot, and improving the transient response. The nominal cases of these control action is shown in the figs $8,9,10,11,12$.

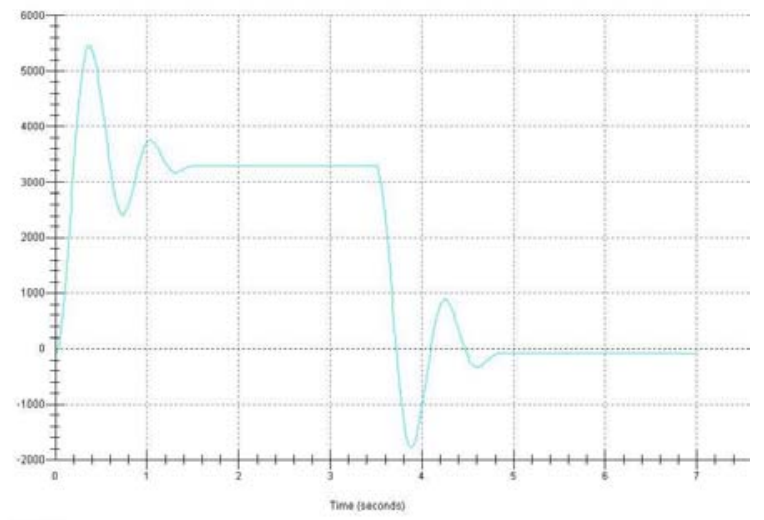

Fig 6 Under-damped system ECP Model 210

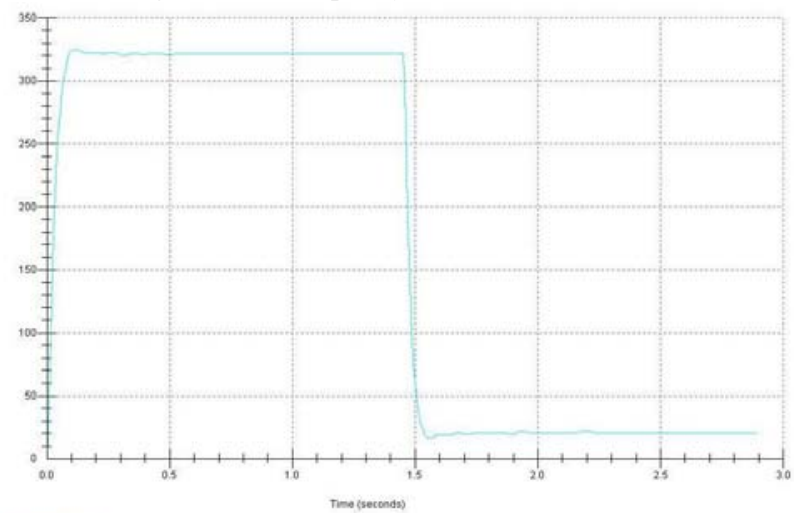

Fig 7 Critically-damped system ECP Model 210

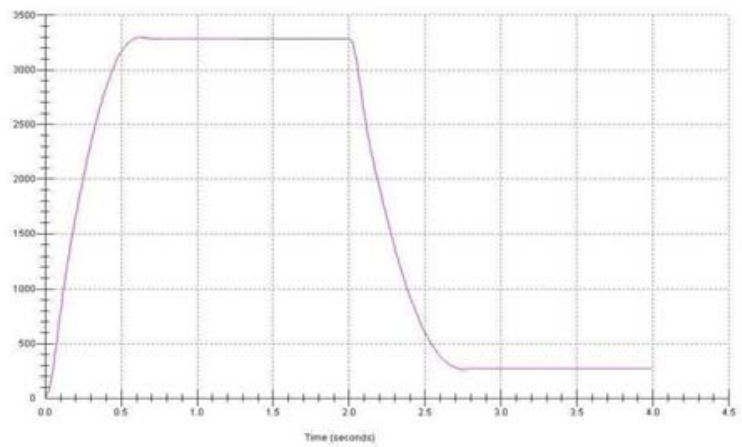

Fig 8 Over-damped system ECP Model 210

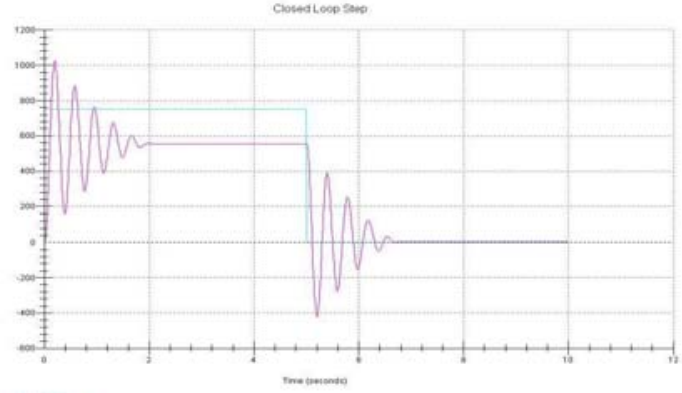

Fig 9 Closed Loop Response- Proportional Control action

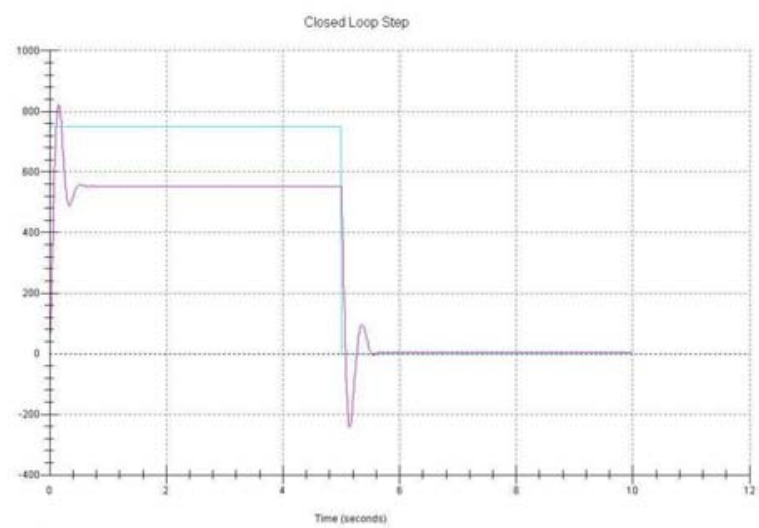

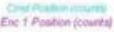

Fig 10 Closed Loop Response- Proportional and Derivative Control Action

cosed loop Step

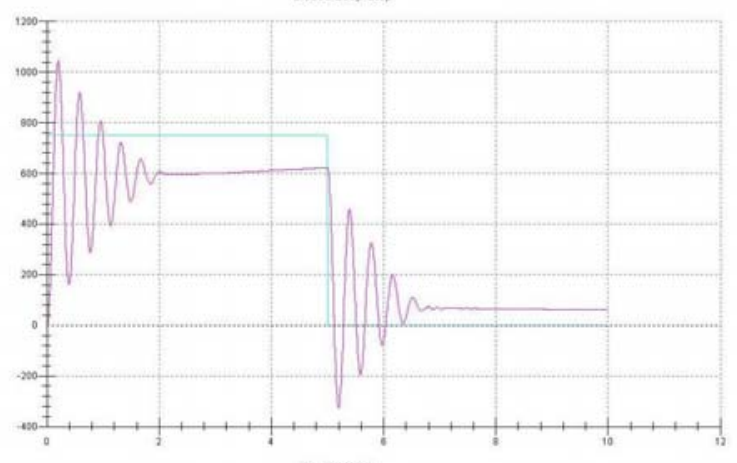

secthentiont

Fig 11 Closed Loop Response- Proportional and Integral Control Action

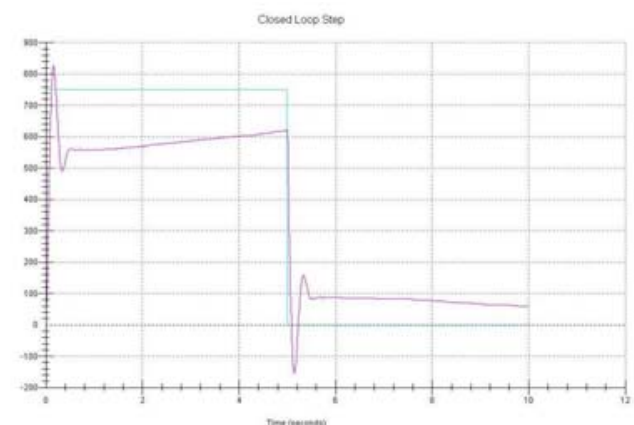

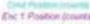

Fig 12 Closed Loop Response-Proportional, Integral and Derivative Control Action 


\section{CONCLUSION}

The Automatic Control Laboratory at the ECE Dept WVU TECH has been successful. The laboratory exercises have coincided with the class materials, providing a visual aid for the lecture concepts. With unstructured instructions students have improved their retention capabilities and reasoning skills.

A sequence of laboratory exercises is presented in this paper that has tried to maximize student involvement in system identification, analysis, design and implementation. The Automatic Control Laboratory has been conducted for a semester and it has established better learning outcomes through the laboratory exercises. The emphasis of unstructured based instructions has come done at the outlay of some facts, such as real-time programming, effect of nonlinearities such as saturation.

Comments from the students:

"For the first time, the labs coincided with what was being taught in class and actually reinforced the class material, providing a visual aid for the lecture concepts. We finally saw why the mathematical models are so important and how they were applied in real-life scenarios. I especially liked the more free-form style of performing the laboratories that this led to". Bridget Meadows Senior electrical engineering student WVU TECH.

"The laboratory equipment I used, under the guidelines of the instructor, greatly enhanced my understanding of control systems". Donald Triplett senior electrical engineering student WVU TECH.

"Personally I think this laboratory was very well taught and informative. This laboratory helped to reinforce some of the class lectures". Lloyd Miranda senior electrical engineering student WVU TECH.

"I found the Controls Lab to be very beneficial to the lecture class. In the lecture class we learn the theory of controls; the lab allows the students to see how the theory becomes practical". Aaron Echard senior electrical and computer engineering student WVU TECH.

\section{ACKNOWLEDGMENT}

A part of the Laboratory equipment is sponsored by West Virginia EPSCoR through instrumentation grant program.

\section{REFERENCES}

[1] Chetan Chandrasekara, Asad Davari "Experiments for the Undergraduate Control Laboratory" $36^{\text {th }}$ IEEE-SSST Proceeding March 2004 Atlanta, Georgia pg 488-491.

[2] Dennis S. Bernstein "What Makes Some Control Problems Hard" Control system Magazine IEEE Vol. 22, issue 4, Aug 2002 pg 8-19.

[3] Armstrong, B. Perez, R. "Controls laboratory program with an accent on discovery learning" Control system Magazine IEEE Vol. 21, issue 1, Feb 2001 pg 14-20.

[4] Bernstein, D. "A plant taxonomy for designing control experiments" Control system Magazine IEEE Vol. 21, issue 3, Jun 2001 pg 7-14.
[5] Thomas R. Parks “ECP Instructor's Manual for Model 210/210a Rectilinear Control System” 1999.

[6] Thomas R. Parks "ECP Instructor's Manual for Model 205/205a Torsional Control System" 1999.

[7] Educational Control Products "ECP Addendum For: User Written Control Algorithm" 1999.

[8] Bonnie S. Heck. "Future Directions in Control Education" Control system Magazine Vol. 19, issue 5, Oct 1999 pg 36-37.

[9] Peter Dorato "Undergraduate Control Education in the U.S." Control system Magazine Vol. 19, issue 5, Oct 1999 pg 38-39.

[10] Bernstein, D.S. "Enhancing Undergraduate Control Education" Control system Magazine Vol. 19, issue 5, Oct 1999 pg 40-43.

[11] Asad Davari and Duoyan Shen "On-Line Control of a Real System with Matlab/Simulink" $30^{\text {th }}$ IEEE-SSST Proceeding March 1998 Morgantown West Virginia pg 7-9.

[12] Asad Davari and Duoyan Shen "Simple and Inexpensive Control Laboratory" $29^{\text {th }}$ IEEE-SSST Proceeding March 1997 Cookeville Tennessee pg 145-147.

[13] E.K.Boukas "Automatic Control Laboratory and Project Course" American Control Conference June 1991 Vol. 1 of 3 Boston Massachusetts pg 318-320.

[14] Feedback Control \& Instrumentation "Manual for Servo Fundamentals Trainer SFT154",

[15] Feedback Control \& Instrumentation "Introduction to Analogue Control ES151". 